\title{
A Fourier Series Proof That the Axial like the Transverse Optical Doppler Shift Impacts Time and Information Rates
}

\author{
Samuel Reich \\ Tribco Inc., Cleveland, OH, USA \\ Email:slrch53@gmail.com
}

How to cite this paper: Reich, S. (2019) A Fourier Series Proof That the Axial like the Transverse Optical Doppler Shift Impacts Time and Information Rates. Journal of High Energy Physics, Gravitation and Cosmology, 5, 992-994.

https://doi.org/10.4236/jhepgc.2019.54054

Received: August 5, 2019

Accepted: September 9, 2019

Published: September 12, 2019

Copyright $\odot 2019$ by author(s) and Scientific Research Publishing Inc. This work is licensed under the Creative Commons Attribution International License (CC BY 4.0).

http://creativecommons.org/licenses/by/4.0/

\begin{abstract}
In writings on relativity time, the various relations are only changed by the transverse shift. This paper proves that the axial Doppler shift does that as well and gives some impacts of that on common differential relations in physics. When a modulated signal lasting a time $=\mathrm{T}$ is subjected to an optical Doppler shift K (either axial or transverse or both), where $\mathrm{K}$ is shifted frequency/original frequency, the Doppler shifted signal will last $\mathrm{T} / \mathrm{K}$. This because all shifted harmonics of its Fourier series (with a fundamental period of $\mathrm{T}$ ) will last $1 / \mathrm{K}$ times the period of the original harmonic. The reader can graph any Fourier series and then graph its shifted series. The reader will see the shifted period is $\mathrm{T} / \mathrm{K}$. The Fourier series of the original repeats when time is greater than $T$ and the shifted one when time is greater than $T / K$, which means the original series only represents the signal from time $=0$ to $\mathrm{T}$ and the shifted series represents the shifted signal from time $=0$ to $\mathrm{T} / \mathrm{K}$. Hence, the shifted one has all of the information in $T / K$ as the original has in $T$. Therefore everything in the series including information is $\mathrm{T} / \mathrm{K}$ long in the shifted series. Therefore, both the axial and the transverse Doppler shift change time periods in a vacuum, independent of material involved. That has not been obvious for over 100 years the axial shift changes time from the definition of frequency $=1 /$ time.
\end{abstract}

\section{Keywords}

Space Time, Relativity, Doppler, Time, Information Rates, Information Transfer, Missing Dimensions

\section{Fourier Series Proof of the Effect of All Doppler Shifts on Modulation in Time Periodic Objects}

Any set of periodic objects or events (including any modulation) observed for a 
length of time $G$ is a piecewise continuous function and therefore has a convergent Fourier series representation. The value of each nth harmonic has the form An $\sin (\mathrm{n} \omega 0 \mathrm{~T}+\mathrm{c})$, where $\omega 0$ is the fundamental frequency and An are constants. The optical Doppler shift multiplies the frequency of each of the harmonics by a factor of $\mathrm{K}$. So the value of a harmonic at time $\mathrm{T}$ now happens at $\mathrm{T} / \mathrm{K}$. Therefore, the value of the sum of all harmonics at $\mathrm{T}$ (by superposition) also now occurs at $\mathrm{T} / \mathrm{K}$. That means that the whole function of a time period $=\mathrm{G}$ now has a period of $\mathrm{G} / \mathrm{K}$, not $\mathrm{G}$. It is left to the reader to verify by calculation that: for $T$ greater than $G$, the values of the Fourier series representation simply repeat the original wave and do the same for values of $T$ greater than $G / K$ in the shifted function or wave. The wave or function has been compressed or dilated in time depending on $\mathrm{K}$ being greater than one or less than one. $\mathrm{K}>1$ in the blue shift and $\mathrm{K}<1$ in the red shift. Note that a repeater (mirror) is moving to both the source and observer, the Doppler shift is K squared of the above.

Therefore, the Doppler shift affects information (modulation) in a signal not just the carrier frequency.

The reader is invited to make a Fourier series of any function, then multiply all the harmonics' frequency by a factor $(\mathrm{K})$, then plot the original series and the shifted series to verify the above by mathematical experiment.

It would be simpler to just say by definition frequency $=1 /$ time period, therefore anything that changes frequency alone changes time. But that has not been obvious in regard to the optical axial Doppler shift for over a hundred years, the proof by Fourier analysis may be more convincing.

\section{How Axial Blue Shift Supplies Some Candidates for Bohm's Missing Dimensions}

\subsection{A Law of Time (For Use on the Blue Shift) [1]}

Exact observations of the future are impossible because of unknown noise or multiple futures. This is because, in a universe with only one future, any group with a future observing device would try to negate undesirable and avoidable events. But in the macroscopic world with only one future, an event and its negation cannot exist at the same time. So that group could not obtain an exact observation of an avoidable and undesirable event until at the very least the event was no longer avoidable. Therefore, all way to get information from the future implies there exist some undocumented noise or multiple futures in the nature of time. First, confirm that you have the correct template for your paper size.

\subsection{Problems with Information in Blue Shifted Carrier Waves Reflected from a Repeater [1]}

If a modulated beam of duration $\mathrm{G}$ is reflected by a repeater (a mirror or other object absorbs it before sending a repeat) back to the source, the source sees a Doppler shift factor not just $\mathrm{K}$ but $\mathrm{K}^{2}$. The existence of Doppler radar proves 
that reflections by a moving mirror have a Doppler shift. When a repeater moves toward both the source and the observer, the observer sees $\mathrm{K}^{2}$ a double blue shift. This means, if there is no built-in noise and there is only one future, information would be seen faster by the observer than it is sent by the source. That is the observer seeing information coming from the future. But from the new law of time, an observer on the source cannot see an avoidable event until the event is no longer avoidable.

The existence of time travel ideas such as worm holes also has the same problems for the same reason. Since information can be sent by Morse code with bursts of noise, noise can be eliminated as an explanation. As said before, multiple time dimensions are at odds with the common concept of time. But the blue shift implies such dimensions. David Bohm proposed a long time ago that the probabilistic nature of quantum mechanics implies missing dimensions. The axial Doppler blue shift supplies candidate dimensions for the missing dimensions.

\subsection{Schrodinger Time Varying Equation with Multiple Time Dimensions

$$
i \hbar \mathrm{d} \psi / \mathrm{d} t=H \psi \quad \text { (Hamiltonian of the mass) }
$$

For many time dimensions, let ${ }^{j} t$ be the value of the $f^{\text {th }}$ time dimension and ${ }^{m} \Psi$ the wave function (probability of the value of ${ }^{w} t$ ). Then obviously for a great number of $j$ s: you use an integral: $i h \int\left(\partial^{j}\left({ }^{w} \psi\right) / \partial t^{j}\right) \mathrm{d} j={ }^{w} H^{w} \psi$. For a few $j \mathrm{~s}$ you use a sum: $i \hbar \sum^{j} \partial^{j}{ }^{w} \psi / \partial t^{j}={ }^{w} H^{w} \psi$, see references [1] and [2] for more application of the results of this paper.

\section{Conflicts of Interest}

The author declares no conflicts of interest regarding the publication of this paper.

\section{References}

[1] Reich, S.L. and Perera, W.G. (2019) The Difference in Mass between Relativity and Quantum Mechanics, Also Novel Effects of the Axial Doppler Shift. Journal of High Energy Physics, Gravitation and Cosmology, 5, 629-637. https://doi.org/10.4236/jhepgc.2019.53035

[2] Reich, S.L. (2019) Effect of Doppler Shifts on Photon and Particle Flux of Beams, also Light Beam Energy Flux. Researchgate.net.

https://doi.org/10.13140/RG.2.2.32485.55527 